\title{
Relationship between urinary zearalenone concentration and embryo production in superovulated cattle
}

\author{
Mitsuhiro Takagi', Toshiya Hirai ${ }^{3}$, Satoshi Shiga', Seiichi Uno², Emiko Kokushi², Takeshige \\ Otoi $^{4}$, Eisaburo Deguchi ${ }^{1}$, Chenga Tshering ${ }^{5}$ and Johanna Fink-Gremmels ${ }^{6}$ \\ 'Laboratory of Farm Animal Production Medicine, Faculty of Fisheries, Kagoshima University, Kagoshima, Japan, \\ ${ }^{2}$ Faculty of Fisheries, Kagoshima University, Kagoshima, Japan, ${ }^{3}$ Genetics Hokkaido Association, Hokkaido, Japan, \\ ${ }^{4}$ Laboratory of Animal Reproduction, Yamaguchi University, Yamaguchi, Japan, ${ }^{5}$ National Institute of Animal Health of \\ Bhutan, Bhutan, ${ }^{6}$ Faculty of Veterinary Medicine, Utrecht University, Utrecht, Netherlands
}

\begin{abstract}
This field study aimed to investigate the relationships between the urinary zearalenone (ZEN) concentration, which reflects dietary ZEN intake, and the numbers of total and transferable embryos in superovulated cattle. A total of 38 cows (Japanese Black, $n=16$; Holstein, $n=22$ ) were superovulated for commercial embryo production. Urine samples were collected from all cows at the time of embryo flushing and the urinary ZEN concentration was measured. The ZEN concentration was corrected for the creatinine (Crea) concentration as follows: ZEN $(\mathrm{pg} / \mathrm{mL}) / \mathrm{Crea}(\mathrm{mg} / \mathrm{dL})$; the corrected ZEN concentration was expressed in $\mathrm{pg} / \mathrm{mg}$ Crea. The cows were divided into two groups according to whether the urinary ZEN level was less than (group 1) or more than (group 2) the mean value for each breed (Japanese Black: $97.4 \mathrm{pg} / \mathrm{mg}$ Crea; range 44.5-91.3 pg/mg Crea; Holstein: $155.5 \mathrm{pg} / \mathrm{mg}$ Crea; range $32.7-146.9 \mathrm{pg} / \mathrm{mg}$ (rea). The embryo flushing results were compared between the two groups within each breed. Overall, the total number of embryos collected and the number of transferable embryos did not differ significantly between the groups. These results suggest that natural ZEN contamination resulting in urine levels below the threshold value (i.e. below the maximal permissible urinary ZEN concentration) does not affect embryo production in Japanese Black and Holstein cows undergoing superovulation.
\end{abstract}

Keywords: cow, embryo production, urine, zearalenone

Abrreviations: ZEN: zearalenone, Crea: creatinine

Archiv Tierzucht 56 (2013) 36, 360-366

Received: 26 September 2012

doi: 10.7482/0003-9438-56-036

Accepted: 20 December 2012

Corresponding author:

Online: 22 March 2013

Mitsuhiro Takagi; email: mtakagi@agri.kagoshima-u.ac.jp

Laboratory of Farm Animal Production Medicine, Kagoshima University, Kagoshima 890-0064, Japan

() 2013 by the authors; licensee Leibniz Institute for Farm Animal Biology (FBN), Dummerstorf, Germany.

This is an Open Access article distributed under the terms and conditions of the Creative Commons Attribution 3.0 License (http://creativecommons.org/licenses/by/3.0/). 


\section{Introduction}

Embryo production by superovulation in cattle has been used for commercial beef and dairy production since the 1970s and remains the fundamental method for producing in vivo-derived embryos for embryo transfer in cattle (Takagi et al. 2001, Velazquez 2011). Although bovine superovulation programmes are now relatively well-established (Bo et al. 2008), the yield and quality of the embryos may be affected by donor factors such as age, breed, reproductive and lactation status, gonadotropin preparation and nutrition (Hasler et al. 1983, Donaldson \& Ward 1986, Takagi et al. 2001, Donaldson 1984, Lerner et al. 1986). The embryo yield and quality may also be affected by feed toxicants such as mycotoxins (McEvoy et al. 2001).

Zearalenone (ZEN) is a non-steroidal estrogenic mycotoxin produced by Fusarium species. This mycotoxin is found in the grains of several monocotyledons including pasture grasses. Zearalenone and its metabolites exert agonistic effects on the oestrogen receptor and thus exhibit distinct oestrogenic and anabolic properties with varying effects on the reproductive system in several animal species particularly pigs (Kleinova et al. 2002, FinkGremmels \& Malekinejad 2007, Minervini \& Dell'Aquila 2008). Binder et al. (2007) recently reported a relatively high incidence of contamination of animal feeds with mycotoxins that could potentially affect animal production. However, the relevance of these high levels is difficult to assess. Several reports have suggested that dietary intake of ZEN is associated with reproductive problems such as infertility, reduced milk production and hyperoestrogenism in cattle (Weaver et al. 1986, Coppock et al. 1990, D'Mello et al. 1999, Minervini \& Dell'Aquila 2008). However, to the best of our knowledge, there is no published report of the relationship between the yield and quality of the embryos obtained after induction of superovulation and the daily level of ZEN intake from naturally ZEN-contaminated feeds on a cattle farm. The concentration of ZEN in the urine of farm animals such as cattle is presumed to be a biomarker for ZEN exposure (Prelusky et al. 1989, Usleber et al. 1992, Kleinova et al. 2002). Furthermore, we recently reported that monitoring the ZEN concentration in the urine of cattle that are fed naturally ZEN-contaminated feed by using an ELISA-based method is an effective way of estimating daily ZEN intake (Takagi et al. 2011).

The objective of this preliminary study was to investigate the relationships between the urinary ZEN concentrations of cows under farm conditions and the number of total and transferable embryos collected after superovulation.

\section{Materials and methods}

\section{Chemicals and solvents}

Ammonium acetate and high-performance liquid chromatography (HPLC)-grade methanol were purchased from Wako Pure Chemicals (Osaka, Japan). Beta-Glucuronidase/arylsulfatase solution was purchased from Merck (Darmstadt, Germany). Sodium acetate was purchased from Kanto Chemical Co., Inc. (Tokyo, Japan) and Tris was purchased from Nakalai Tesque, Inc. (Kyoto, Japan).

\section{Animals}

Thirty-eight apparently healthy cows (Japanese Black [n=16] and Holstein [n=22]) from 25 farms in Hokkaido (Japan) were subjected to superovulation for commercial embryo production. 
The average age was $4.3 \pm 2.8$ years for the Japanese Black animals and $5.2 \pm 3.2$ years for the Holstein animals. Although the details of the dietary management of each cow examined on the farm were not available for the present study, the Japanese Black and Holstein cows were generally fed in accordance with the Japanese Feeding Standard for Japanese Black breeding cows and the Japanese Feeding Standard for Holstein cows, respectively and all cows had free access to fresh water. In addition, the mean body condition score on a scale of 1 (too thin) to 5 (obese) of each cow was determined by a skilled veterinarian in order to assess the energy balance. All cows examined in the present study scored between 3.0 and 3.5.

\section{Embryo production}

Superovulation was induced after following the method of Bo et al. (1995) with a minor modification. Superovulation was initiated mid-cycle (8-12 days after oestrus) and a total of 20 AU or 40 AU of follicle-stimulating hormone (FSH; Antrin, Kyoritsu Seiyaku Corp., Tokyo, Japan) was administrated intramuscularly over 8 injections of decreasing doses at 12-h intervals to the Japanese Black and Holstein cows, respectively. On the third day, a prostaglandin F2a analogue (PGF2a; Pronalgon F, Pfizer, Tokyo, Japan) was administered to induce regression of the corpus luteum. The cows underwent artificial insemination approximately 60 to $72 \mathrm{~h}$ after PGF2a administration.

Seven days after artificial insemination, the embryos were flushed from the uteri of donor cows with phosphate-buffered saline supplemented with foetal bovine serum and antibiotics. One single skilled veterinarian performed all of the flushes in the study. Immediately after collection, the embryos were assessed for viability using a stereomicroscope. The embryos were also graded according to their stages of development and quality (transferable, degenerated and unfertilized) as defined by previously described criteria (Robertson \& Nelson 1998). Briefly, embryos in which more than $25 \%$ of the total cell mass was considered viable and development was not severely retarded were considered transferable. The evaluation records of the recovered embryos were used in the present study.

\section{Urine sample collection}

Urine samples were collected immediately after individual embryo recovery during natural urination stimulated by soft massage of the pudendum. The urine samples were immediately stored in a light-proof cooler and transported to the laboratory. The samples were then centrifuged at $500 \times \mathrm{g}$ for $10 \mathrm{~min}$ and the supernatants frozen at $-30^{\circ} \mathrm{C}$ until analysis.

\section{Analytical methods for determination of ZEN and creatinine concentrations}

As described in detail in our previous report (Takagi et al. 2011), the concentration of ZEN in urine was determined using a commercially available kit (RIDA SCREEN Zearalenon, R-Biopharm AG, Darmstadt, Germany) according to the manufacturer's instructions with minor modifications. The mean recovery rate of the ELISA assay over the 3 trials was $84 \pm 14 \%$. The ZEN analysis results were not corrected for the recovery rate.

Urinary Crea concentrations were determined using a commercial kit (Sikarikit-S CRE, Kanto Chemical) according to the manufacturer's instructions and were measured with a clinical autoanalyzer (7700 Clinical Analyzer, Hitachi High-Tech, Tokyo, Japan). In the present 
study, the urinary ZEN concentration is expressed relative to the urinary Crea concentration (urinary ZEN/urinary Crea [pg/mg Crea]) as previously described (Takagi et al. 2011).

\section{Statistical Analysis}

The results are presented as the mean \pm SEM. The relationships between the urinary ZEN concentrations and embryo production results within the breed groups were evaluated using Pearson's correlation coefficient. The percentages of transferable embryos (number of transferable embryos/number of collected embryos) were also compared between the two groups using the unpaired t-test. Statistical analyses were carried out using the STATVIEW (Abacus Concepts, Inc., Berkeley, CA, USA) programme. Differences with a probability value $(P)$ of 0.05 or less were considered significant.

\section{Results}

No significant correlations were observed between the urinary ZEN concentration and embryo parameters neither in Japanese Black cows (urinary ZEN concentration vs. total number of recovered embryos, $r=-0.2$ and $P=0.96$ and urinary ZEN concentration vs. transferable embryo rate, $r=0.2$ and $P=0.48$ ) or Holstein cows (urinary ZEN concentration vs. total number of recovered embryos, $r=0.1$ and $P=0.82$ and urinary $Z E N$ concentration vs. transferable embryo rate, $r=-0.4$ and $P=0.87$ ).

The cows of each breed were divided into two groups on the basis of the respective mean urinary ZEN concentrations of the Japanese Black (97.4 pg/mg Crea) and Holstein (155.5 pg/ $\mathrm{mg}$ (rea) cows in the present study. Group 1 consisted of 11 Japanese Black cows (44.5$91.3 \mathrm{pg} / \mathrm{mg}$ Crea) and 14 Holstein cows (32.7-146.9 pg/mg (rea) and group 2 consisted of 5 Japanese Black cows (103.8-226.0 pg/mg Crea) and 8 Holstein cows (201.0-404.5 pg/mg Crea). No significant differences in the numbers of total embryos collected, transferable embryos, degenerated embryos or unfertilized embryos were observed between the two groups within each breed (Table 1).

Table 1

Relationships between the urinary ZEN concentration and the yield and quality of embryos (mean \pm SEM) collected after induction of superovulation in cows

\begin{tabular}{|c|c|c|c|c|c|}
\hline Group* & $\begin{array}{l}\text { No. of } \\
\text { cows }\end{array}$ & $\begin{array}{c}\text { Total } \\
\text { embryos (No.) }\end{array}$ & $\begin{array}{l}\text { Transferable } \\
\text { embryos, } \%\end{array}$ & $\begin{array}{l}\text { Degenerated } \\
\text { embryos, } \%\end{array}$ & $\begin{array}{l}\text { Unfertilized } \\
\text { embryos, \% }\end{array}$ \\
\hline \multicolumn{6}{|l|}{ Japanese Black } \\
\hline \multicolumn{6}{|l|}{ Group 1} \\
\hline $\begin{array}{l}\text { (<97.4 pg/mg of Crea) } \\
\text { Group } 2\end{array}$ & 11 & $16.4 \pm 2.6$ & $7.8 \pm 1.7(48.9)$ & $6.5 \pm 2.2(33.6)$ & $2.1 \pm 0.6(17.6)$ \\
\hline (>97.4 pg/mg of Crea) & 5 & $13.8 \pm 4.1$ & $10.8 \pm 3.7(63.1)$ & $2.4 \pm 1.3(15.2)$ & $0.6 \pm 0.4(21.7)$ \\
\hline \multicolumn{6}{|l|}{ Holstein } \\
\hline \multicolumn{6}{|l|}{ Group 1} \\
\hline ( $<155.5 \mathrm{pg} / \mathrm{mg}$ of Crea) & 14 & $13.4 \pm 2.0$ & $9.6 \pm 1.4(71.9)$ & $2.9 \pm 1.0(17.4)$ & $0.8 \pm 0.5(10.7)$ \\
\hline \multicolumn{6}{|l|}{ Group 2} \\
\hline (>155.5 pg/mg of Crea) & 8 & $13.0 \pm 5.3$ & $8.4 \pm 4.2(58.8)$ & $3.5 \pm 1.1(32.1)$ & $1.1 \pm 0.6(9.2)$ \\
\hline
\end{tabular}

* Japanese Black and Holstein cows were divided into two groups on the basis of the mean ZEN concentration for each breed obtained from the present study. 


\section{Discussion}

Recently, we reported that exposure to ZEN via naturally contaminated feed can be monitored at the farm level by measuring urinary ZEN concentrations by using an ELISAbased method (Takagi et al. 2011, Hasunuma et al. 2012). We found this monitoring system to be effective for the objective assessment of dietary ZEN intake under different sets of farm conditions. The Ministry of Agriculture, Forestry and Fisheries in Japan has set a maximum ZEN level of $1000 \mu \mathrm{g} / \mathrm{kg}(1 \mathrm{ppm})$ for formula feeds. Although we cannot compare the total ZEN concentrations between our previous and present studies because of possible differences in the breeds and body weights of the cattle, our previous observation of the relationship between urinary and dietary ZEN concentrations (Hasunuma et al. 2012) has led us to assume that all cows examined in the present study received feeds with levels of ZEN permissible under the current guidelines (less than $1 \mathrm{ppm}$ ). Our present results, which were obtained using our established urinary ZEN monitoring system under general and practical farm conditions, indicated that at the farm level under typical feeding conditions the ovarian response/embryo production after induction of superovulation in cows might not be affected by feeds containing permissible levels of ZEN.

Although cattle are not as sensitive to ZEN as pigs, several reports have described a negative effect of ZEN and its metabolites on the reproductive performance of cattle (Diekman \& Green 1992, McEvoy et al. 2001). Feeding ZEN to dairy heifers ( $250 \mathrm{mg} /$ day) over 3 oestrous cycles decreased the conception rate from 87 to $62 \%$ (Weaver et al. 1986) and pastureacquired ZEN has been implicated in the development of infertility in cattle in New Zealand (Towers \& Sprosen 1993). On the other hand, Chorfi et al. (2007) reported that ZEN at a natural dietary concentration (174.1 $\pm 120 \mathrm{ppb}$ ) did not significantly affect the number of transferable embryos after superovulation. However, this level might not reflect the actual dietary intake of ZEN. Previous observations of the effect of ZEN on reproductive performance in cattle may be contradictory because of the huge variations in the amounts of ZEN administered orally in the different experiments or because the ZEN concentrations examined were not derived from biological samples of individual animals. In the present study, the cows were divided according to their urinary ZEN levels into two groups and parameters such as numbers of totally collected and transferable embryos were compared between these groups. The results of the present study suggest that neither the quantity nor the quality of embryos collected after ovarian stimulation by standard commercial methods is affected by dietary ZEN intake under normal farm conditions. The present study did not include counting the numbers of follicles present during and after the superovulation/flushing treatments. Therefore, a further study using daily ultrasound observation of the ovaries will be required to clarify the effect of the urinary ZEN concentration on both follicular development and ovulation after induction of superovulation.

In conclusion, the results of our field trials suggest that the dietary intake of ZEN as estimated indirectly by the urinary ZEN concentration does not significantly affect the numbers of total and transferable embryos obtained after induction of superovulation in cows. Further studies employing ultrasonographic examination to investigate the relationship between dietary ZEN intake from normal feed and follicular development during induction of superovulation are warranted. 


\section{Acknowledgements}

This study was supported by a Grant-in-Aid for Scientific Research (No. 20580355 to M.Takagi) from the Japan Society for the Promotion of Science (JSPS).

\section{References}

Binder EM, Tan LM, Chin LJ, Handl J, Richard J (2007) Worldwide occurrence of mycotoxins in commodities, feeds and feed ingredients. Anim Feed Sci Technol 137, 265-282

Bo GA, Adams GP, Pierson RA, Mapletoft RJ (1995) Exogenous control of follicular wave emergence in cattle. Theriogenology 43, 31-40

Bó GA, Guerrero DC, Adams GP (2008) Alternative approaches to setting up donor cows for superstimulation. Theriogenology 69, 81-87

Chorfi Y, Lanevschi A, Dupras R, Girard V, Tremblay A (2007) Serum biochemical parameters and embryo production during superovulatory treatment in dairy cattle. Res Vet Sci 83, 318-321

Coppock RW, Mostrom MS, Sparling CG, Jacobsen B, Ross SC (1990) Apparent zearalenone intoxication in a dairy herd from feeding spoiled acid-treated corn. Vet Hum Toxicol 32, 246-248

Diekman MA, Green ML (1992) Mycotoxins and reproduction in domestic livestock. J Anim Sci 70, 1615-1627

Donaldson LE, Ward DN (1986) Effects of luteinising hormone on embryo production in superovulated cows. Vet Rec 119, 625-626

Donaldson LE (1984) Cattle breed as a source of variation in embryo transfer. Theriogenology 21, 1013-1018

D'Mello JPF, Placinta CM, Macdonald AMC (1999) Fusarium mycotoxins: a review of global implications for animal health, welfare and productivity. Anim Feed Sci Technol 80, 183-205

Fink-Gremmels J, Malekinejad H (2007) Clinical effects and biochemical mechanisms associated with exposure to the mycoestrogen zearalenone. Anim Feed Sci Technol 137, 326-341

Hasler JF, McCauly AD, Schermerhorn EC, Foote RH (1983) Superovulatory responses of Holstein cows. Theriogenology 19, 83-99

Hasunuma H, Takagi M, Kawamura O, Taniguchi C, Nakamura M, Chuma T, Uno S, Kokushi E, Matsumoto D, Tshering C, Deguchi E, Fink-Gremmels J (2012) Natural contamination of dietary rice straw with zearalenone and urinary zearalenone concentrations in a cattle herd. J Anim Sci 90, 1610-1616

Kleinova M, Zöllner P, Kahlbacher H, Hochsteiner W, Lindner W (2002) Metabolic Profiles of the Mycotoxin Zearalenone and of the Growth Promoter Zeranol in Urine, Liver, and Muscle of Heifers. J Agric Food Chem 50, 4769-4776

Lerner SP, Thayne WV, Baker RD, Henschen T, Meredith S, Inskeep EK, Dailey RA, Lewis PE, Butcher RL (1986) Age, Dose of FSH and Other Factors Affecting Superovulation in Holstein Cows. J Anim Sci 63, 176-183

McEvoy TG, Robinson JJ, Ashworth CJ, Rooke JA, Sinclair KD (2001) Feed and forage toxicants affecting embryo survival and fetal development. Theriogenology 55, 113-129

Minervini F, Dell'Aquila ME (2008) Zearalenone and Reproductive Function in Farm Animals. Int J Mol Sci 9, 2570-2584

Prelusky DB, Warner RM, Trenholm HL (1989) Sensitive analysis of the mycotoxin zearalenone and its metabolites in biological fluids by high-performance liquid chromatography. J Chromatogr B Biomed Sci Appl 494, 267-277

Robertson I, Nelson RE (1998) Certification and identification of the embryo. In: Stringfellow DA, Seidel SM (eds.) Manual of the International Embryo Transfer Society, 3rd rev. ed., Urbana, IL, USA, 103-134

Takagi M, Kim IH, Izadyar F, Hyttel P, Bevers MM, Dieleman SJ, Hendriksen PJM, Vos PLAM (2001) Impaired final follicular maturation in heifers after superovulation with recombinant human FSH. Reproduction 121, 941-951 
Takagi M, Uno S, Kokushi E, Shiga S, Mukai S, Kuriyagawa T, Takagaki K, Hasunuma H, Matsumoto D, Okamoto K, Shahada F, Deguchi E, Fink-Gremmels J (2011) Measurement of urinary zearalenone concentrations for monitoring natural feed contamination in cattle herds: On-farm trials. J Anim Sci 89, 287-296

Towers NR, Sprosen JM (1993) Zearalenone-induced infertility in sheep and cattle in New Zealand. N Z Vet J 41, 223-224

Usleber A, Renz V, Märtlbauer E, Terplan G (1992) Studies on the Application of Enzyme Immunoassays for the Fusarium Mycotoxins Deoxynivalenol, 3-Acetyldeoxynivalenol, and Zearalenone. J Vet Med B 39, 617-627

Velazquez MA (2011) The role of nutritional supplementation on the outcome of superovulation in cattle. Anim Reprod Sci 126, 1-10

Weaver GA, Kurtz HJ, Behrens JC, Robison TS, Seguin BE, Bates FY, Mirocha CJ (1986) Effect of zearalenone on the fertility of virgin dairy heifers. Am J Vet Res 47, 1395-1397 